\title{
Future Challenges of Flow Measurement: towards smart and compact sensors
}

\author{
W. Drahm, O. Popp, M. Touzin, Endress+Hauser GmbH+Co. KG, Freising, Germany
}

Flowmeters, instruments for the flow measurement of gases, liquids and solids, are today based on a variety of mechanical, electronic, or electro-mechanical principles.

How has the business of flow measurement developed over the years? The first widely used and commercially available flowmeters were mechanical, and were developed over 100 years ago. Since these "traditional" technologies still today make up a significant part of the market, one could speak of the "evolution" of flow measurement. Around 60 years ago, electronic flowmeter technologies were introduced and have also evolved. Many millions of flowmeters are manufactured and sold every year by over 1000 companies, and are installed in hundreds of different applications in dozens of industry sectors.

The value of the industrial flowmeter market for closed pipe gas and liquid applications is shared roughly 50/50 between 4 traditional technologies (which include Positive Displacement, Turbine, Variable Area and Differential Pressure methods), and 5 newer, primarily electronic technologies (Electromagnetic, Vortex, Coriolis, Thermal and Ultrasonic). The bulk of the industrial flowmeter business today is comprised of these 9 broad categories, each of which has sub-categories which were developed and exist because they meet customer needs. Another dozen technologies could be added if those for special niche applications were included.

Based on consensus market research, we know that although traditional flowmeters still represent a significant part of the market, the market growth rate of these technologies is much slower than that for electronic flowmeters. Substitution is taking place, evidenced by the high growth rate of modern technologies.

In industrial process applications, flow measurement plays an important role in ensuring product quality and process safety, and in determining the value of transferred fluids.To be successful, flowmeter manufacturers must listen closely to their customers to understand what developments and instrument characteristics will bring added value. Added value means different things to different people, depending on what is important for the specific process industry and for the specific application in that industry. Using the principle of "fit for purpose", the optimum combination of technology, price, measurement performance, and functionality resulting in the best choice for a given task can easily be determined.

What does the future hold for users of industrial flowmeters? Modern electronic flowmeter technologies are continuously evolving,resulting in added value in several areas for end users. For example, the need to ensure reliable, long-term in-process measurement performance has resulted in a variety of self-diagnosis techniques to ensure measurement validity. Endress + Hauser's Heartbeat ${ }^{(\mathrm{TM})}$ technology for flowmeters, for example, is a big step in this direction. Modular, platform-based manufacturing, enables users to "build" flowmeters which are basic or advanced, depending on the mechanical and electronic needs of the application, such asflowmeter size, price, materials, approvals, power requirements, signal transmission, or measurement performance.

ThePromass 100 Coriolis flowmeter, for example, has a very compact design, for applications where space is at a premium, but with built-in verification diagnostics and digital communication. Last but not least, "multi-parameter" measurement capability, or the ability to simultaneously determine additional measured or derived process variables, can result in better process understanding and control. The Prosonic Flow B200 ultrasonic flowmeter, designed for biogas flow measurement, also simultaneously determines the percentage of methane in the gas.

Flow measurement technologies will continue to evolve in future, reflecting new and unmet customer needs. 